\section{Corticosteroid- induced intraocular pressure elevation in keratoconus is common following uncomplicated penetrating keratoplasty}

JC Fan, K Chow, DV Patel and CNJ McGhee
Faculty of Medical and Health Sciences, Department of Ophthalmology, University of Auckland, Auckland, New Zealand

Correspondence: CNJ McGhee, Faculty of Medical and Health Sciences, Department of Ophthalmology, University of Auckland, Private Bag 92019, Auckland 1,

New Zealand

Tel: +6493737599

Ext 86712;

Fax: + 6493677173

E-mail: c.mcghee@

auckland.ac.nz

Received: 3 June 2008 Accepted in revised form: 1 December 2008 Published online: 20 March 2009

\begin{abstract}
Aims To determine the incidence of postkeratoplasty intraocular pressure (IOP) elevation in the eyes of subjects with keratoconus and establish the relationship between IOP and corticosteroid administrations in this population. Methods Following strict inclusion/exclusion criteria, a retrospective analysis was performed on a consecutive series of penetrating keratoplasties performed for keratoconus observing a standardised surgical and postoperative regimen in Auckland, New Zealand. Patient demographics, ocular, medical, and family history, and pre- and postoperative data were recorded until 12 months postkeratoplasty.

Results In all, 57 eyes of 48 patients were included-31\% New Zealand Europeans, 42\% Pacific people, $15 \%$ Maori, and $12 \%$ other. Eighteen eyes $(32 \%)$ of 17 patients $(35 \%)$ exhibited elevated IOP and $12(21 \%)$ eyes exhibited moderate-to-severe elevation of IOP. IOP elevation occurred 3-6 months postkeratoplasty in $78 \%$ of eyes. Elevated IOP was significantly less common in Maori and Pacific peoples $(P=0.02)$. All eyes except one required reduction/cessation of corticosteroids to normalise IOP.

Conclusions The incidence of presumed steroid-related postkeratoplasty IOP elevation, in $35 \%$ of subjects with keratoconus, is markedly higher in this New Zealand study than previously reported in the US and UK studies. Further clinical and genetic analysis of associations between keratoconus and
\end{abstract}

steroid-induced IOP elevation and glaucoma might improve our current understanding of this condition.

Eye (2009) 23, 2056-2062; doi:10.1038/eye.2008.413; published online 20 March 2009

Keywords: keratoconus; penetrating keratoplasty; intraocular pressure; corticosteroids

\section{Introduction}

Intraocular pressure (IOP) elevation is a known complication of topical corticosteroid therapy and was first described by Francois in $1954 .^{1}$ Those who are likely to respond generally do so within a few weeks of continual steroid administration. ${ }^{1}$ Steroid-induced pressure response in children tends to be more prevalent and rapid than adults. ${ }^{2}$ In both adults and children, however, IOP typically returns to normal following cessation of steroid therapy.

Postpenetrating keratoplasty IOP control is a major concern for ophthalmic surgeons. Not only can prolonged IOP elevation damage the optic nerve, ${ }^{3}$ it can be harmful to the donor endothelium and ultimately affect the success of the corneal graft. ${ }^{4}$ It is also among the more difficult of the glaucomas to monitor due to the effect of significant astigmatism on accurate applanation measurement of IOP, particularly in the early postoperative period. ${ }^{5}$ Treatment of this elevated IOP is also challenging, as prolonged topical corticosteroid therapy is an inherent part of postkeratoplasty management.

In Australasia, keratoconus is the most common indication for penetrating 
keratoplasty. ${ }^{6,7}$ Studies that have examined elevation of IOP following penetrating keratoplasty have reported the incidence to be in the range of $10-35 \% ;^{3,8-11}$ however, keratoconus subjects have been noted to be among the least at risk to encounter this complication. ${ }^{3,12,13}$ Yet, in contradistinction from clinical experience, we suspected that a higher proportion of the keratoconus population in New Zealand experienced elevated IOP postpenetrating keratoplasty than previously reported in the world literature. This study was, therefore, undertaken to determine the incidence of postpenetrating keratoplasty IOP elevation in keratoconus as well as to establish the relationship between IOP and topical corticosteroid administrations in this population.

\section{Materials and methods}

\section{Patient selection}

This study used a retrospective study design to assess subjects referred for penetrating keratoplasty for keratoconus. Analysis was restricted to the records of all penetrating keratoplasties performed by one corneal subspecialist surgeon (CNJM) for keratoconus during the period of January 2000 to March 2008 at the Department of Ophthalmology, Auckland City Hospital and Eye Institute, Auckland, New Zealand. A standardised assessment, surgical technique, and postoperative topical corticosteroid regime were used throughout the study period.

The exclusion criteria included deep anterior lamellar keratoplasty, repeat penetrating keratoplasty, any previous intraocular surgery, history of glaucoma, any additional surgery within the follow-up period, and tertiary referrals from outside the region who were unable to attend regular local follow-up. Three patients who were lost to follow-up during the study period were also excluded.

Patient demographic information, ocular and medical history, including any previous use of topical or systemic corticosteroids, and family history of keratoconus and/or glaucoma were recorded. Operative information recorded included endothelial density of donor cornea, type of sutures used, and intraoperative drug administration. Postoperative antibiotic and corticosteroid regime was standardised. Postoperative records were followed to 12 months postkeratoplasty for all patients and those (eight subjects) who had not reached this point of follow-up were excluded.

IOP measurements by Goldmann's tonometer were taken routinely for each visit and were recorded for each of the following visits (or closest thereof): preoperative assessment, 1-week postoperatively, 1, 3, 6, 9, and 12 months. Elevated IOP before 1-week postsurgery was excluded to avoid inadvertently including viscoelasticrelated IOP elevation.

For the purpose of analysis, normal IOP was defined as $10-21 \mathrm{mmHg}$. Elevated IOP between $22-25 \mathrm{mmHg}$ was considered as 'mild', 26-30 mmHg as 'moderate', and measurements $>30 \mathrm{mmHg}$ were considered as 'severe' IOP elevation.

Any interventions, for example, reduction in frequency/potency of corticosteroids or introduction of topical ocular hypotensives, in response to elevation of IOP rise were recorded. The subsequent change in IOP in response to these interventions was noted. The total number of drops (cumulative dose) of topical corticosteroid administered during the entire postoperative period was determined from each individual patient record. Corneal topography with central corneal thickness measurements was also recorded during the postoperative period.

\section{Surgical technique and postoperative management}

Penetrating keratoplasty was performed using a BarronHessberg suction trephine system (Barron Precision Instruments, LLC, MI, USA). The donor material was excised from the endothelial side by using a BarronHessberg donor punch system (Barron Precision Instruments) and all donor tissue was sized $0.25 \mathrm{~mm}$ larger in trephine diameter than the host (excepting one case that was deliberately oversized by $0.50 \mathrm{~mm}$ ). The standard suturing technique used 12 interrupted 10/0 nylon sutures and an antitorque continuous $11 / 0$ nylon except when significant peripheral corneal neovascularisation was encountered - in which setting 16 interrupted 10/0 nylon sutures were utilised. In all eyes, a viscoelastic substance (Healon, Advanced Medical Optics Inc., Santa Ana, CA, USA) was used to form the anterior chamber and protect the endothelium during surgery. Just before the completion of surgery, this material was aspirated or washed out of anterior chamber and the anterior chamber was completely reformed with balanced salt solution and the water tightness of the wound confirmed.

All patients received subconjunctival dexamethasone sodium phosphate $(2 \mathrm{mg}$ in $0.5 \mathrm{ml}$ ) and cephazolin $(100 \mathrm{mg}$ in $0.5 \mathrm{ml}$ ) injections at the end of the procedure. All subjects were prescribed three doses of oral acetazolamide $250 \mathrm{mg}$ in the first $18-24 \mathrm{~h}$ after surgery.

Postoperative management included g. chloramphenicol four times a day (Chlorsig, Sigma Pharmaceuticals) for 2-4 weeks and g. prednisolone acetate 1\% (Predforte, Alcon Laboratories) at approximately 2-h intervals during waking hours for the initial 7 postoperative days, then four times a day for 6 months, reducing stepwise to once per day for months 
9-12. Eight patients were subsequently placed on g. dexamethasone $0.1 \%$ to reduce medication cost (g. prednisolone acetate $1 \%$ is not a governmentsubsidised drug in New Zealand, whereas g. dexamethasone $0.1 \%$ is subsidised) or g. fluoromethalone $0.1 \%$, if a less potent corticosteroid was required in the context of a steroid-related IOP elevation to g. prednisolone acetate. The cumulative number of corticosteroid drops applied per patient was counted based on prescribed regime for the 12-month follow-up.

\section{Statistical analysis}

Statistical analyses were performed in conjunction with a professional biomedical statistician. To investigate factors related to the occurrence of raised IOP after surgery, a generalised mixed model was fitted to allow adjustment for the within person correlation for those who had two eyes operated on. The binary outcome of elevated IOP or not was used with the logit link function, and age, gender, and ethnicity as explanatory variables, whereras theuse of topical and systemic steroids, IOP, and family history of keratoconus preoperatively, and postoperative central corneal thickness were considered as explanatory variables.

\section{Results}

In a largely tertiary referral practice, a total of 269 keratoplasties were performed by the one surgeon over an 8-year period, including 257 penetrating and 12 deep lamellar keratoplasties. Of the penetrating keratoplasties, 87 were performed for keratoconus; 64 for regraft purposes; 36 for keratitis; 22 for pseudophakic bullous keratopathy; 20 for corneal dystrophies; 12 for trauma, and 16 were conducted for other indications. Of the 89 primary penetrating keratoplasties conducted for keratoconus, 17 were excluded as they had not reached the 12-month postsurgical follow-up period; three were excluded due to previous intraocular procedures; one was excluded because the penetrating keratoplasty was performed in conjunction with vitrectomy; and nine were either transferred back to the referring centre or lost to follow-up.

Therefore 57 eyes of 48 patients who met the exclusion and inclusion criteria were included in this study. The male-to-female ratio was 29:19. Right-to-left eye ratio was $22: 35$. In all, $31 \%$ of patients were New Zealand Europeans, 25\% Samoan, 17\% other Pacific people, 15\% Maori, $6 \%$ Indian, $4 \%$ other European, and 2\% were Middle Eastern. None of the patients had an earlier ocular history of elevated IOP or glaucoma.
A total of $75 \%$ of subjects did not have any family history of glaucoma, and in $23 \%$, this was unknown. Only one patient had a known family history of glaucoma in a first-degree relative, and this subject did not have a family history of keratoconus. In all, $19 \%$ of eyes had a history of previous topical corticosteroid use; in all cases, this had been used in the treatment of an episode of acute hydrops. Seventy-nine percentage of eyes had not had previous topical corticosteroid use, and this information was unknown in $2 \%$, whereas $25 \%$ had previous systemic corticosteroid use in the form of asthma inhalers or oral corticosteroids for asthma; $67 \%$ had not had previous systemic steroids, and in $8 \%$ this was unknown.

The median age of patients undergoing penetrating keratoplasty in this study was 29 years (range: 13-59). The mean \pm SD preoperative best-corrected Snellen visual acuity was $3 / 36$ (mean logMAR $0.78 \pm 0.49$ ). Reliable preoperative IOP was available for 42 eyes (74\%), and the mean was $12.5 \pm 2.8 \mathrm{mmHg}$. Because of the severity of disease, only $25 \%$ of eyes had reliable preoperative central corneal thickness measurement available on Orbscan II topography despite attempts of recording this on all patients; in these cases, the mean central corneal thickness was $391.07 \pm 88.12 \mu \mathrm{m}$. The first available mean central corneal thickness between 6 weeks and 12 months after surgery was $503.2 \pm 80.8 \mu \mathrm{m}$; this was unavailable in 18 eyes. Subjects applied topical corticosteroids (of any preparation) for a mean number of $315 \pm 65$ days (range: 118-365).

Eighteen eyes (32\%) of seventeen patients (35\%) had elevated IOP at some point during the 12-month followup period (Table 1) - one at 1 month, nine eyes at 3 months, five at 6 months, one at 9 months, and two at 12 months (median: 3 months). Of these patients, nine were New Zealand Europeans, two other Europeans, two Indians, one Maori, and three Pacific people. The male-to-female ratio was 12:5. The median age at the time of penetrating keratoplasty of this group was 33.5 years (range: 18-59). Six of these eyes had mildly elevated IOP, seven had moderately elevated, and five had severely elevated IOP (ie, $12(21 \%)$ of 57 eyes had moderate-to-severe elevation of IOP). The highest recorded IOP was $50 \mathrm{mmHg}$ in a 20-year-old Tongan man at the 3-month postkeratoplasty follow-up. The pressure was lowered immediately with topical ocular antihypertensives as well as oral acetazolamide. This enabled the IOP to drop to $13 \mathrm{mmHg}$ within $3 \mathrm{~h}$. However, the patient required topical ocular antihypertensives throughout the rest of the postoperative period until topical steroids were stopped. Four other subjects recorded IOP peaks of greater than $30 \mathrm{mmHg}$, being 32, 32, 34, and $34 \mathrm{mmHg}$, respectively. Formal perimetry was not performed during the first 
Table 1 Postkeratoplasty pressure elevation over 12-month follow-up in keratoconus

\begin{tabular}{lcccccc}
\hline & \multicolumn{5}{c}{ Number of eyes } \\
\cline { 2 - 7 } & 1 week & 1 month & 3 months & 6 months & 9 months & 12 months \\
\hline Mild $(22-26 \mathrm{mmHg})$ & 0 & 0 & 3 & 1 & 1 & 1 \\
Moderate $(27-31 \mathrm{mmHg})$ & 0 & 1 & 3 & 2 & 0 & 1 \\
Severe $(>31 \mathrm{mmHg})$ & 0 & 0 & 3 & 2 & 0 & 0 \\
\hline
\end{tabular}

Table 2 Treatment methods used to treat elevated IOP from 1-week to 12-month postkeratoplasty

\begin{tabular}{lc}
\hline Treatment methods for elevated IOP & $\begin{array}{c}\text { Number of } \\
\text { eyes }\end{array}$ \\
\hline $\begin{array}{l}\text { Reduction or cessation of corticosteroids alone } \\
\text { Addition of ocular antihypertensive alone }\end{array}$ & 5 \\
$\begin{array}{l}\text { Addition of ocular antihypertensive initially, but } \\
\text { reduction of corticosteroids required }\end{array}$ & 4 \\
$\begin{array}{l}\text { Addition of ocular antihypertensive and reduction } \\
\text { of corticosteroids simultaneously }\end{array}$ & 7 \\
$\begin{array}{l}\text { Reduction of corticosteroids, addition of ocular } \\
\text { antihypertensive and oral acetazolamide } \\
\text { simultaneously }\end{array}$ & 1 \\
\hline
\end{tabular}

12 months postkeratoplasty in those with transiently elevated IOP; however, clinically no optic disc damage was identified.

If only the first operated eye for each patient was considered, the incidence of elevated IOP was 33\% (16 eyes of 48 patients). Only two patients with bilateral keratoplasty had elevated IOP postsurgically. The first patient had elevated IOP in both eyes. In this case, the keratoplasties were 1 year apart, and the first eye developed mildly elevated IOP $(23 \mathrm{mmHg})$, whereas the second eye suffered severely elevated IOP $(32 \mathrm{mmHg})$. Elevation of IOP in each eye was first noted at 6-month postkeratoplasty. The second patient experienced raised IOP only in the second eye to undergo corneal transplantation. The operations were 13 months apart for this subject, and elevated IOP $(28 \mathrm{mmHg})$ was detected only at the 12-month postsurgery clinic review.

Table 2 outlines the treatment methods used to normalise IOP in those with elevated pressures from 1 week to 12 months postkeratoplasty, including $39 \%$ of eyes requiring simultaneous addition of an ocular antihypertensive as well as reduction of topical corticosteroids; $28 \%$ requiring reduction or cessation of topical corticosteroids alone; $22 \%$ received treatment by addition of topical ocular antihypertensives initially, but reduction of corticosteroids was necessary later.

None of the patients required surgical management of elevated IOP. None of the eyes required ongoing treatment with an ocular antihypertensive agent once the
IOP normalised and the corticosteroids were withdrawn. None of the eyes showed IOP elevation during the follow-up period once topical corticosteroid was stopped.

Comparing those who experienced elevated IOP to those who did not, the IOP responders were less likely to be of Maori or Pacific ethnicity, and this was statistically significant $(P=0.02)$. However, no statistical difference was found when comparing age $(P=0.89)$, gender $(P=0.96)$, history of previous ocular $(P=0.33)$ or systemic $(P=0.82)$ steroid administration, family history of keratoconus $(P=0.40)$, preoperative IOP $(P=0.53)$, continuous and interrupted sutures or interrupted sutures only $(P=0.26)$, or postoperative central corneal thickness $(P=0.59)$.

The mean cumulative number of steroid drops at the point of IOP rise for the 18 eyes with elevated IOP was 630.1 ( \pm 346.5$)$. The mean 12-month cumulative steroid dose for the IOP responders was $929.8( \pm 310.3)$, and for the non-IOP-responders, it was 1228.2 ( \pm 338.1 ; $P=0.003$ - due to cessation or reduction of topical corticosteroids in the IOP responder group. None of the eyes experienced allograft rejection as a result of the reduction of corticosteroids, and specifically, rejection did not occur more frequently in those whose corticosteroids were reduced due to elevated IOP $(P=0.185)$.

\section{Discussion}

In this study, we examined the occurrence of IOP elevation in patients undergoing penetrating keratoplasty for keratoconus. To the authors' knowledge, this is the first study to closely examine elevated IOP postkeratoplasty in a large cohort of keratoconic subjects selected to exclude other risk factors.

This study confirms our suspicions that the incidence of postkeratoplasty-elevated IOP is substantial between 1 week and 12 months postkeratoplasty at $32 \%$, with $11 \%$ of eyes developing mild, $12 \%$ moderate, and $9 \%$ severe IOP elevation. The incidence of this potentially deleterious effect of topical corticosteroids is significantly higher than that reported previously for keratoconus (see Table 3). Part of the discrepancy may be due to the differences in the inclusion and exclusion criteria 
Table 3 Comparison of percentage of postkeratoplasty-elevated IOP in keratoconus with other studies

\begin{tabular}{|c|c|c|c|}
\hline Author & Country & $\begin{array}{l}\text { Number of grafts } \\
\text { for keratoconus }\end{array}$ & $\begin{array}{l}\text { Percentage of keratoconic } \\
\text { eyes with elevated IOP (\%) }\end{array}$ \\
\hline Goldberg et $a l^{11}$ & USA & 20 & 15 \\
\hline Kirkness and Moshegov ${ }^{14}$ & UK & 99 & 12 \\
\hline Simmons et $a^{13}$ & USA & 40 & 10 \\
\hline Kirkness and Ficker ${ }^{3}$ & UK & 202 & 2 \\
\hline Sihota $e t a l^{8}$ & India & 26 & 4 \\
\hline Fan $e t a l^{2}$ & New Zealand & 57 & 32 \\
\hline
\end{tabular}

between the studies. For example, in the study conducted by Kirkness and Ficker, ${ }^{3}$ steroid responders were excluded from the study as they were examining persistent elevated IOP following keratoplasty; Goldberg et $a l^{11}$ defined elevated IOP as being $25 \mathrm{mmHg}$ or higher, whereas our criteria for elevated IOP started at $22 \mathrm{mmHg}$. However, even excluding those with mildly elevated IOP, this study still shows a higher incidence (21\%) for moderate to severe elevation of IOP postkeratoplasty. Potential confounders in earlier studies included the inclusion of significant number of subjects who received combined surgery or eyes that were aphakic; however, as they did not differentiate these factors by diagnosis of corneal condition, a direct comparison cannot be made with this study as to whether these variables altered the outcome of IOP in keratoconus.

Other than corticosteroid-induced IOP elevation, several factors have previously been reported to be associated with late elevation in IOP postkeratoplasty; these include preoperative glaucoma or elevated IOP, ${ }^{12,13}$ aphakia, ${ }^{8}$ penetrating keratoplasty combined with anterior segment reconstruction and anterior vitrectomy, ${ }^{3,8,13}$ and repeat penetrating keratoplasty. ${ }^{11}$ As this study excluded all of these co-morbid conditions as possible confounders and concentrated on subjects with keratoconus in isolation, the factors most likely to be responsible for elevating the IOP in these subjects are penetrating keratoplasty per se or the IOP response to corticosteroids. However, as all eyes except one required treatment modification by reduction or cessation of steroids (with or without topical ocular antihypertensives) to normalise IOP, and subsequently no eyes showed elevated IOP following cessation of corticosteroids, a steroid-related elevation of IOP seems the most likely mechanism in this study. With half of the subjects having developed elevated IOP by 3 months postoperatively, the timing of the IOP elevation is also consistent with corticosteroid-induced ocular hypertension, which usually occurs within a few weeks of treatment with potent corticosteroids, or within months with less potent steroids. ${ }^{1}$
Corticosteroid-induced ocular hypertension is an incompletely understood phenomenon. Most evidence suggests that the cause of corticosteroid-induced hypertension is a reduction in outflow of aqueous fluid through the trabecular meshwork in the anterior chamber of the eye in susceptible individuals. ${ }^{15}$ Risk factors postulated to be associated with steroid response, some shared with those for postkeratoplasty IOP elevation, include a history of glaucoma or glaucoma suspect, ${ }^{16}$ older age, ${ }^{14}$ and previous steroid response. Whereas $18-36 \%$ of the general population have previously been found to have an increase of $5 \mathrm{mmHg}$ or more in IOP after topical corticosteroids, ${ }^{15,17} 5-6 \%$ of the general population and $46-92 \%$ of patients with primary open-angle glaucoma experience significant and potentially damaging rise in IOP after topical steroids administration. Therefore, although the overall proportion of steroid responders per se in this study is not higher than what is reasonable to encounter by chance, the percentage of patients with a moderate or severe IOP rise is much higher.

It is perhaps surprising that earlier studies assessing PKP in keratoconus have not shown the same level of steroid responsiveness in their study populations. Kirkness and Moshegov ${ }^{14}$ found that only $5 \%$ of all eyes had raised IOP that responded to manipulation of steroids. As previously noted, Kirkness and Ficker ${ }^{3}$ excluded all steroid responders, and many other studies have failed to comment on steroid-induced IOP elevation at all.

Comparing patients who developed postoperative IOP elevation to those who did not, the only factor that could be significantly correlated with IOP elevation was ethnicity. The Caucasian subjects of European origin were much more likely to develop postoperative IOP elevation compared with Maori and Pacific peoples in this study. This is surprising as one might assume that ethnicities unique to New Zealand might account, at least in part, for the significantly higher incidence of IOP elevation in this study compared with the established studies primarily conducted in Europe and in the United States. 
Little has been published on aqueous humour dynamics in keratoconus; Goodman et $a^{18}$ found that there was no difference in mean aqueous flow rates in keratoconus compared with controls, and proposed that the lower mean IOP in this population is due to increased outflow facility. This could explain the lower rate of IOP elevation after keratoplasty in keratoconus in other studies, but helps little to explain the current observations. As it is well established that there is a genetic basis to both keratoconus and primary openangle glaucoma, ${ }^{19,20}$ one must consider the possibility that the keratoconic population in New Zealand may represent a distinct genotype that exhibits a linkage between the gene(s) responsible for glaucoma and those responsible for the development of steroid-induced ocular hypertension.

Although an individual may be considered as either a steroid-responder or not, the cumulative dose of corticosteroids required to 'turn on' the steroid responsiveness varies from individual to individual and possibly between eyes. This may explain why, in the current series, one patient with identical bilateral penetrating keratoplasty procedures and identical postoperative steroid regimens only experienced elevated IOP in the second eye to undergo surgery. Interestingly, however, following the 12-month closure date of the current study, at 18-month postkeratoplasty, the subject developed significant elevation of IOP in the first eye during an episode of graft rejection and treatment with intensive hourly prednisolone acetate drops.

The mean 12-month cumulative steroid dose was significantly lower for those with elevated IOP than those without IOP elevation because the patient was much more likely to have their corticosteroid dose reduced due to IOP elevation than if the IOP remained normal. Although there is no 'routine' postoperative topical corticosteroid regime that is widely accepted in the published literature, ${ }^{8,12,21}$ our routine management for all postcorneal transplant cases is gutte prednisone acetate $1 \%$ QDS for 6 months. Although it has been well established that long-term topical steroid treatment improves graft survival following normal risk penetrating keratoplasty, ${ }^{22}$ those with reduced corticosteroids in this cohort were not found to be more likely to develop graft rejection during the study period. However, as it has been suggested that steroid responders may be at higher risk of developing glaucoma later in life, ${ }^{23,24}$ glaucoma screening in this population is advisable. Future genetic studies into this group of patients may help confirm our postulation.

In conclusion, this study has revealed a higher incidence of postkeratoplasty IOP elevation in keratoconus subjects than previously reported, yet the populations unique to this study, Maori and Pacific peoples, were associated with a lower likelihood of this complication. Although no direct relationship between total dosage of topical corticosteroid and IOP elevation after keratoplasty was identified, all but one of the 18 eyes that experienced elevated IOP regained normal pressure once the topical corticosteroid was reduced or stopped. Given that elevated IOP may not only affect the success of the corneal transplant but also cause glaucomatous optic neuropathy, further clinical and genetic studies into the links between keratoconus, steroid-induced ocular hypertension and glaucoma might improve the current understanding and optimise clinical management of this condition.

\section{Acknowledgements}

We thank Joanna Stewart (biostatistician), and Shalina Navaratnam (medical student) for their input into this study.

\section{References}

1 Francois J. Cortisone et tension oculaire. Ann D'Oculist 1954; 187: 805.

2 Kwok AK, Lam DS, Ng JS, Fan DS, Chew SJ, Tso MO. Ocular-hypertensive response to topical steroids in children. Ophthalmology 1997; 104: 2112-2116.

3 Kirkness CM, Ficker LA. Risk factors for the development of postkeratoplasty glaucoma. Cornea 1992; 11: 427-432.

4 Charlin R, Polack FM. The effect of elevated intraocular pressure on the endothelium of corneal grafts. Cornea 1982; 1: 241-249.

5 Irvine AR, Kaufman HE. Intraocular pressure following penetrating keratoplasty. Am J Ophthalmol 1969; 68: 835-844.

6 Edwards M, Clover GM, Brookes N, Pendergrast D, Chaulk $\mathrm{J}$, McGhee CN. Indications for corneal transplantation in New Zealand: 1991-1999. Cornea 2002; 21: 152-155.

7 The Australian Corneal Graft Registry. 1990 to 1992 Report. Aust N Z J Ophthalmol 1993; 21: 1-48.

8 Sihota R, Sharma N, Panda A, Aggarwal HC, Singh R. Post-penetrating keratoplasty glaucoma: risk factors, management and visual outcome. Aust N Z J Ophthalmol 1998; 26: 305-309.

9 Wilson SE, Kaufman HE. Graft failure after penetrating keratoplasty. Surv Ophthalmol 1990; 34: 325-356.

10 Chien AM, Schmidt CM, Cohen EJ, Rajpal RK, Sperber LT, Rapuano CJ et al. Glaucoma in the immediate postoperative period after penetrating keratoplasty. Am J Ophthalmol 1993; 115: $711-714$

11 Goldberg DB, Schanzlin DJ, Brown SI. Incidence of increased intraocular pressure after keratoplasty. Am J Ophthalmol 1981; 92: 372-377.

12 Jonas JB, Rank RM, Hayler JK, Budde WM. Intraocular pressure after homologous penetrating keratoplasty. J Glaucoma 2001; 10: 32-37.

13 Simmons RB, Stern RA, Teekhasaenee C, Kenyon KR. Elevated intraocular pressure following penetrating 
keratoplasty. Trans Am Ophthalmol Soc 1989; 87: 79-91, (discussion 91-3)

14 Kirkness CM, Moshegov C. Post-keratoplasty glaucoma. Eye 1988; 2(Suppl): S19-S26.

15 Armaly MF. Effect of corticosteroids on intraocular pressure and fluid dynamics. Ii. the effect of dexamethasone in the glaucomatous eye. Arch Ophthalmol 1963; 70: 492-499.

16 Becker B, Mills DW. Corticosteroids and intraocular pressure. Arch Ophthalmol 1963; 70: 500-507.

17 Becker B. Intraocular pressure response to topical corticosteroids. Invest Ophthalmol 1965; 4: 198-205.

18 Goodman WT, Mathers WD, Munden PM, Ossoinig KC, Daley TE. A study of aqueous humor dynamics in keratoconus. Exp Eye Res 1996; 62: 95-99.

19 Edwards M, McGhee CN, Dean S. The genetics of keratoconus. Clin Experiment Ophthalmol 2001; 29: 345-351.

20 Wiggs JL. Genetic etiologies of glaucoma. Arch Ophthalmol 2007; 125: 30-37.
21 Seitz B, Langenbucher A, Nguyen NX, Kuchle M, Naumann GO. Long-term follow-up of intraocular pressure after penetrating keratoplasty for keratoconus and Fuchs' dystrophy: comparison of mechanical and Excimer laser trephination. Cornea 2002; 21: 368-373.

22 Nguyen NX, Seitz B, Martus P, Langenbucher A, Cursiefen C. Long-term topical steroid treatment improves graft survival following normal-risk penetrating keratoplasty. Am J Ophthalmol 2007; 144: 318-319.

23 Lewis JM, Priddy T, Judd J, Gordon MO, Kass MA, Kolker $\mathrm{AE}$ et al. Intraocular pressure response to topical dexamethasone as a predictor for the development of primary open-angle glaucoma. Am J Ophthalmol 1988; 106: 607-612.

24 Kitazawa Y, Horie T. The prognosis of corticosteroidresponsive individuals. Arch Ophthalmol 1981; 99: 819-823. 